\title{
Frühe Herzinsuffizienz Beide Geschlechter profitieren von einer Phytotherapie
}

\author{
Wenn die Herzleistung bei Belastung \\ nachlässt, hat sich Weißdorn als phyto- \\ therapeutischer Ansatz bewährt.
}

Die Wirkung von Weißdornextrakt spiegelt sich sowohl in der subjektiven Beschwerdeerleichterung als auch in objektiven physiologischen Parametern wider. Dabei spielt das Geschlecht der Patienten für den Behandlungserfolg keine Rolle. Dies zeigt eine Metaanalyse der gepoolten Daten von 687 Patienten mit leichter Herzinsuffizienz, die an placebokontrollierten Studien mit Crataegus-Extrakt teilgenommen hatten. In den Studien wurde überwiegend der standardisierte Extrakt WS 1442 der Firma Schwabe verwendet.

Überprüft wurde der Einfluss der Behandlung auf die maximale Belastungsfähigkeit, die linksventrikuläre Ejektionsfraktion (LVEF) sowie die Zunahme des Druck-Herzfrequenz-Produkts bei einer ergometrischen Belastung von 50 Watt. Alle drei physiolo-

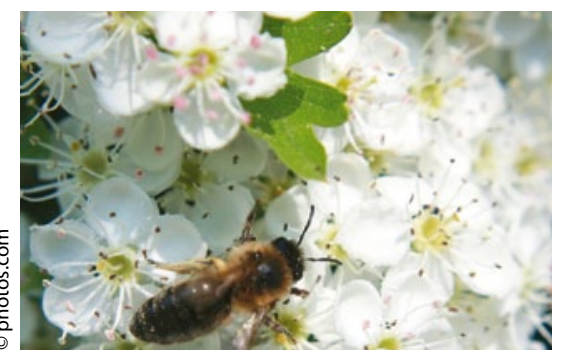

Weißdornextrakt bessert die Symptome einer leichten Herzinsuffizienz.

gischen Parameter besserten sich unter dem Einfluss des Phytopharmakons stärker als unter Placebo. Das Ausmaß der Besserung war unabhängig vom initialen LVEF-Wert, korrelierte jedoch mit den beiden anderen Parametern für den Schweregrad der Erkrankung zu Beginn der Studie.

Auch in Bezug auf typische Symptome wie eingeschränkte Belastungstoleranz, Dyspnoe bei Belastung, Schwäche, Müdigkeit und Palpitationen war der Behandlungser- folg in der Verumgruppe bei Patienten mit anfangs ausgeprägteren Beschwerden größer. Unterschiede zwischen Männern und Frauen erklärten sich hingegen ausschließlich aus dem unterschiedlichen Schweregrad der Erkrankung.

Erhärtet werden die Befunde durch Beobachtungen aus einer dreijährigen Kohortenstudie mit WS 1442 (366 Patienten). Die am häufigsten genannten körperlichen Symptome besserten sich um $40-50 \%$, und dies überwiegend in den ersten sechs Behandlungsmonaten. Die Belastungstoleranz nahm signifikant zu, am stärksten bei den Patienten mit den niedrigsten Ausgangswerten. Allerdings weisen die Autoren darauf hin, dass es sich ausschließlich um Patienten mit nur leicht beeinträchtigter LVEF (im Mittel $43 \%$ ) gehandelt hatte.

(ee) II

II Eggeling T et al. Phytomedicine 2011; doi:10.1016/j.phymed.2011,06022

\section{Typ-2-Diabetes}

\section{Helfen moderne Insuline, die Kostenlawine zu bremsen?}

\section{Die Zahl der Diabetespatienten steigt unaufhörlich - und damit auch die Kosten für das Gesundheitswesen. Für die Kostenbegrenzung ist es vor allem wichtig, Komplikationen des Diabetes zu vermeiden. Davon sind Experten überzeugt.}

Der Anteil der Diabetiker in Deutschland ist in den Jahren 2000 bis 2009 um fast $50 \%$ gestiegen, belegen aktuelle Analysen. Obwohl sich die diabetesbedingten Exzesskosten pro Patient kaum erhöht haben, lassen die steigenden Erkrankungszahlen die direkten Kosten für den Diabetes in diesem Zeitraum um $60 \%$ auf über 20 Milliarden Euro steigen. Über $40 \%$ dieser Ausgaben betreffen stationäre Behandlungen oder Pflegeleistungen. Mikro- und makrovaskuläre Komplikationen sind die Hauptkostentreiber, betonte Dr. med. Andreas Liebl vom Diabetes- und Stoffwechselzentrum der m\&i-
Fachklinik, Bad Heilbrunn. Es sei Zeit, gegenzusteuern: „Die Kostenlawine der Komplikationen wird uns sonst überrollen!"

Die Kosteneffektivität moderner Therapien wird bewertet, indem man deren langfristigen Nutzen gegen die Kosten aufrechnet. In der Regel geschieht dies in Form von QALY, qualitätsadjustierten Lebensjahren, erläuterte der Experte. Als kosteneffektiv gilt z.B. die intensive Blutzuckersenkung. Kalkuliert auf der Basis der UKPD-Studie kostet ein durch intensive Glukosekontrolle gewonnenes QALY rund 41000 US-Dollar, berichtete Liebl.

Moderne Analoga wie Insulin aspart (NovoRapid ${ }^{\oplus}$ ) erlauben nach seiner Ansicht eine besonders kosteneffektive Therapie. Als Beleg führte er z.B. eine pharmaökonomische Berechnung auf Basis einer randomisierten japanischen Endpunktstudie an. In der Studie hatten 374 Typ-2-Diabetespatienten eine Therapie mit Insulin aspart oder
Humaninsulin erhalten. Nach 4,5 Jahren war die Rate kardiovaskulärer Ereignisse unter dem modernen Insulin um $43 \%$ geringer als unter dem Humaninsulin (11,1 vs. $6,4 \%)$, was vor allem auf die verringerten postprandialen Blutzuckerspitzen, die als kardiovaskulärer Risikofaktor gelten, zurückgeführt wurde.

Für japanische Verhältnisse errechnet sich daraus, dass bereits ab dem zweiten Therapiejahr die Einsparungen durch Vermeidung der Komplikationen größer sind als die zusätzlichen Ausgaben für das Analogon. Für Deutschland ist dies sogar noch günstiger, betonte Liebl. Denn durch die Rabattverträge ist Insulin aspart für $99 \%$ der GKV-Versicherten kostengleich zum Humaninsulin.

(sob) II

II SpringerMedizin Verlagsworkshop „Diabetes und Folgekosten", München, 23. Mai 2012 (unterstützt von Novo Nordisk) 